\title{
Spirals in protoplanetary disks from photon travel time ${ }^{\star}$
}

\author{
M. Kama, P. Pinilla, and A. N. Heays
}

\author{
Leiden Observatory, PO Box 9513, 2300 RA Leiden, The Netherlands \\ e-mail: mkama@strw.leidenuniv.nl
}

Received 13 May 2016 / Accepted 12 August 2016

\begin{abstract}
Spiral structures are a common feature in scattered-light images of protoplanetary disks, and of great interest as possible tracers of the presence of planets. However, other mechanisms have been put forward to explain them, including self-gravity, disk-envelope interactions, and dead zone boundaries. These mechanisms explain many spirals very well, but are unable to easily account for very loosely wound spirals and single spiral arms. We study the effect of light travel time on the shape of a shadow cast by a clump orbiting close (within $\sim 1 \mathrm{au}$ ) of the central star, where there can be significant orbital motion during the light travel time from the clump to the outer disk and then to the sky plane. This delay in light rays reaching the sky plane gives rise to a variety of spiral- and arc-shaped shadows, which we describe with a general fitting formula for a flared, inclined disk.
\end{abstract}

Key words. scattering - methods: observational - protoplanetary disks

\section{Introduction}

Spiral arms are often seen in scattered-light images of protoplanetary disks (e.g. Grady et al. 2001; Hashimoto et al. 2011; Boccaletti et al. 2013; Grady et al. 2013; Avenhaus et al. 2014; Benisty et al. 2015; Wagner et al. 2015; Stolker et al. 2016) and there is much interest in understanding their origins, which might be perturbations by giant planets, disk-envelope interactions, instabilities, or other phenomena. We present a new mechanism for creating scattered-light spirals: the finite speed of light can cause a shadow from the inner disk to morph into a spiral, with properties determined by the disk and viewing geometry, and the orbital motion of the shadowing clump. We derive a fitting formula for the general case of a flared, inclined disk.

A common hypothesis for the origin of spiral pressure waves in disks is that they are driven by giant planets (Goldreich \& Tremaine 1979; Rafikov 2002; Muto et al. 2012; Dong et al. 2015), although a good agreement between planetdisk interaction models and observations is still under debate (e.g. Juhász et al. 2015). Alternative explanations for the spiral arms include gravitational instabilities when the disk is sufficiently cold and massive (e.g. Lodato \& Rice 2004), shocks between a disk and a potential inflow from an external envelope (Lesur et al. 2015), or turbulent waves propagating into dead zones (Lyra et al. 2015).

Some disks display a time-variable scattered-light brightness, which is considered evidence for shadowing by inner disk structures on short orbits (e.g. HD 163296 and J160421, Garufi et al. 2014; Pinilla et al. 2015). Variable inner disk extinction also explains several types of variation in light curves of highly inclined disk-hosting stars such as AA Tau (Bouvier et al. 2007) and a number of sources in the NGC 2264 cluster (McGinnis et al. 2015; Stauffer et al. 2015). In particular, there is a class of sources with regular, narrow dips in their light curves. The inner disk features causing such dimmings could cast azimuthally narrow shadows across the outer disk.

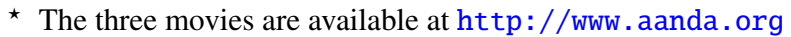

The finite speed of light leads to the propagation of light echoes from time-variable sources such as supernovae and outbursting stars. Light echoes on a scale of $10000 \mathrm{au}$ have been resolved around young stars (Ortiz et al. 2010). Protoplanetary disks themselves, with scattered-light radii of $\sim 100$ to 1000 au (Burrows et al. 1996; McCaughrean \& O'dell 1996; Padgett et al. 1999; McCaughrean et al. 1998; Grady et al. 2001; Wisniewski et al. 2008), are large enough for travel time to play a role in the propagation of light from the central star. Spatially unresolved light travel time effects in a disk on $\sim 100$ au scales were identified in a multi-wavelength time-resolved but spatially unresolved study by Meng et al. (2016), who detected a time delay in back-scattering from the far side of a disk (reverbation).

Light travel time has deeper consequences for resolved imaging. For example, the time for a photon (or, equivalently, a shadow) to travel to a distance $r=100 \mathrm{au}$ at the speed of light $c$ is 0.58 days. In this amount of time, a clump orbiting at 0.1 au from a $1.5 M_{\odot}$ star traverses 0.38 radians or $6 \%$ of its orbit. Combined with the effect of inclination and a radially varying disk surface height, such a shadow can take a wide range of curved shapes. In this Letter, we model the shape of such features. We note that the same curvature applies to bright features cast by a dip in the height of an otherwise azimuthally uniform rim. For conciseness, we use the term "shadow" in the rest of the paper.

In Sect. 2, we present a general model and fitting formula for the of shape of spirals due to light travel time. In Sect. 3, we discuss the observational evidence and implications, and we present our conclusions in Sect. 4.

\section{Spiral features due to photon travel time}

We formulate equations for light travel time spirals first for the flat, face-on disk case; and then for the general case of a flared, inclined disk. In discussing the shape of shadows, we consider the set of light rays that simultaneously reach the sky or, equivalently, observer plane. We furthermore assume that all 


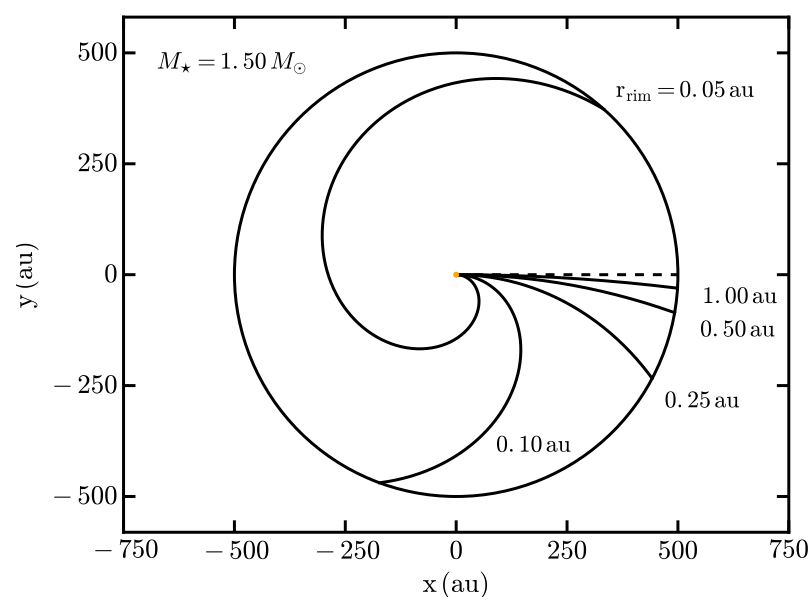

Fig. 1. Spiral-shaped shadows on a flat, face-on disk from light travel time effects. The conical projected shadow (dashed lines) becomes a spiral when finite photon travel times are considered (solid black lines). Spirals from Eq. (1) are plotted for a $1.5 M_{\odot}$ star and a range of shadow origin radii, $r_{\text {rim }}=0.05,0.10,0.25,0.50$, and 1.00 au.

observations are corrected for the disk position angle, such that the semi-minor axis of the disk is vertical, that is pointing North.

\subsection{Flat, face-on disk}

The orbital motion of a shadowing clump, located in the inner disk at the radial location $r_{\text {rim }}$, induces an angular lag $\mathrm{d} \theta_{\text {lag }}(r)=$ $\theta_{\text {rim }}-\theta_{1}$ between the clump and a point along the path of the shadow, with midplane radial location $r_{1}$ :

$\mathrm{d} \theta_{\text {lag }}\left(r_{1}\right)=\left(\frac{r_{1}}{c}\right) \Omega_{\mathrm{K}}\left(r_{\text {rim }}\right)$,

where $\Omega_{\mathrm{K}}\left(r_{\text {rim }}\right)=\left(\mathrm{G} M_{\star}\right)^{0.5} r_{\text {rim }}^{-3 / 2}$ is the Keplerian angular velocity at the orbital location of the shadowing clump, and $M_{\star}$ is the stellar mass. We define $\theta$ to increase in the direction of orbital motion. The dependence of $\mathrm{d} \theta_{\text {lag }}$ on $r_{1}$ describes a spiral, shown in Fig. 1 for several values of the origin radius. As $r_{\text {rim }}$ increases, the shadow will become more straight, and will eventually become a straight line for all reasonable disk sizes.

\subsection{Flared, inclined disk}

We now determine the observed shape of a shadow caused by a clump orbiting in the inner part of an arbitrarily flared and inclined disk. Inclination affects the scattered-light appearance of pressure wave spirals and disk gaps through blocking and reflection of light, leading to non-intuitive shapes (Dong et al. 2016). It also affects light travel time features, as the paths of reflected rays are lengthened or shortened with changing inclination. The scattering surface height $H\left(r_{1}\right)$ is taken to be proportional to the gas scale-height. It generally increases with midplane radius until the very outermost disk, where the surface density drops off and the disk becomes transparent, which we do not include. Adopting the parametric model of Kenyon \& Hartmann (1987), the vertical height from the midplane is

$H\left(r_{1}\right)=h_{\mathrm{c}}\left(\frac{r_{1}}{r_{\mathrm{c}}}\right)^{\psi} r_{1}$,

where $h_{\mathrm{c}}$ is the opening angle corresponding to $H\left(r_{\mathrm{c}}\right)$ as seen from the star, and $\psi$ is the flaring power. The distance $r_{\mathrm{h}}$ that a photon must cross to intersect the disk surface above $r_{1}$ is

$r_{\mathrm{h}}=r_{1}\left(1+\left[h_{\mathrm{c}}\left(\frac{r_{1}}{r_{\mathrm{c}}}\right)^{\psi}\right]^{2}\right)^{1 / 2}$.

For a face-on disk, the increased travel time to the flared surface increases the curvature of the spiral relative to the flat case.

The inclination of a disk adds an extra dimension to light travel time effects, as the light from points along the curve of Eq. (1) no longer reaches the observer at the same time. In midplane polar coordinates, the spiral is specified by the location of the shadowing clump at the time of observing, $\left(\theta_{\text {rim }}, r_{\text {rim }}\right)$, and the midplane location directly under any given point along the observed shadow, $\left(\theta_{1}, r_{1}\right)$. The geometry is illustrated in Fig. 2.

We first consider $t_{r}$, the travel time for a photon from the radial origin of the shadow to a reflecting point on the disk surface, and $t_{z}$, the travel time from that point until a perpendicular intersection with the sky plane at $(z=0)$. The observation of a scattered photon following the $t_{z}+t_{r}$ path will coincide with an observed photon from the inner disk, originating from the clump after time $t_{\Omega}$ in Keplerian rotation and having a light travel time $t_{z, \text { rim }}$ to the sky plane. The four time intervals are

$t_{\Omega}=\frac{\theta_{\text {rim }}-\theta_{1}}{\Omega_{\mathrm{K}}}$,

$t_{z, \text { rim }}=\frac{r_{\text {rim }} \cos \left(\theta_{\text {rim }}\right) \sin (i)}{c}$,

$t_{z}=\frac{r_{1} \cos \left(\theta_{1}\right) \sin (i)-H_{1} \cos (i)}{c}$, and

$t_{r}=\frac{r_{1}-r_{\text {rim }}}{c}$, where $r_{\text {rim }} \leq r_{1}$.

In Eq. (4), we have assumed that the vertical height of the feature casting the shadow has a negligible impact on the light travel time from $r_{\text {rim }}$ to any observable point on the disk surface. Since $t_{\Omega}=\mathrm{d} \theta_{\text {lag }} / \Omega_{\mathrm{K}}$, we can write

$$
\begin{aligned}
\mathrm{d} \theta_{\text {lag }} & =\theta_{\text {rim }}-\theta_{1} \\
& =\Omega_{\mathrm{K}} \times\left(t_{z}+t_{r}-t_{z, \text { rim }}\right) .
\end{aligned}
$$

Solving Eq. (8), assuming $\theta_{\text {rim }} \geq \theta_{1}$ and $r_{\text {rim }} \leq r_{1}$, we find

$r_{1}=r_{\text {rim }} \frac{\left(\theta_{\text {rim }}-\theta_{1}\right) c / v_{\mathrm{K}}+1+\cos \left(\theta_{\text {rim }}\right) \sin (i)}{\left(1+F_{\mathrm{c}}^{2}\right)^{1 / 2}-F_{\mathrm{c}} \cos (i)+\cos \left(\theta_{1}\right) \sin (i)}$,

where $F_{\mathrm{c}}=h_{\mathrm{c}}\left(r_{1} / r_{\mathrm{c}}\right)^{\psi}$ parameterizes the vertical structure. In Fig. 3 we show numerical solutions to Eq. (10) for the flatdisk case $\left(h_{\mathrm{c}}=0\right)$ with $r_{\text {rim }}=0.1$ au for five different inclinations, converted into on-sky $(x, y)$ coordinates according to the equations

$x=r_{1} \sin \left(\theta_{1}\right)$ and

$y=r_{1} \cos \left(\theta_{1}\right) \cos (i)+r_{1} F_{\mathrm{c}} \sin (i)$.

Viewed face-on, the four spirals originating at points $\theta_{\text {rim }}=0$, $\pi / 2, \pi$ and $3 \pi / 2$ rad are all identical. With increasing inclination, their shapes diverge, as the interplay of depth-of-field and Keplerian rotation direction takes effect. The appearance of the spiral is radically different when it is moving towards or away from the observer. For the largest inclinations, the $\theta_{\text {rim }}=\pi \mathrm{rad}$ spiral breaks into two arcs as the middle segment of the spiral falls outside of the outer radius of our model disk.

Figure 3 also shows the calculations for the same inner rim and viewing conditions for a flaring disk $\left(h_{\mathrm{c}}=0.1, \psi=0.25\right.$, $\left.r_{\mathrm{c}}=40 \mathrm{au}\right)$. The three circles correspond to the disk midplane 


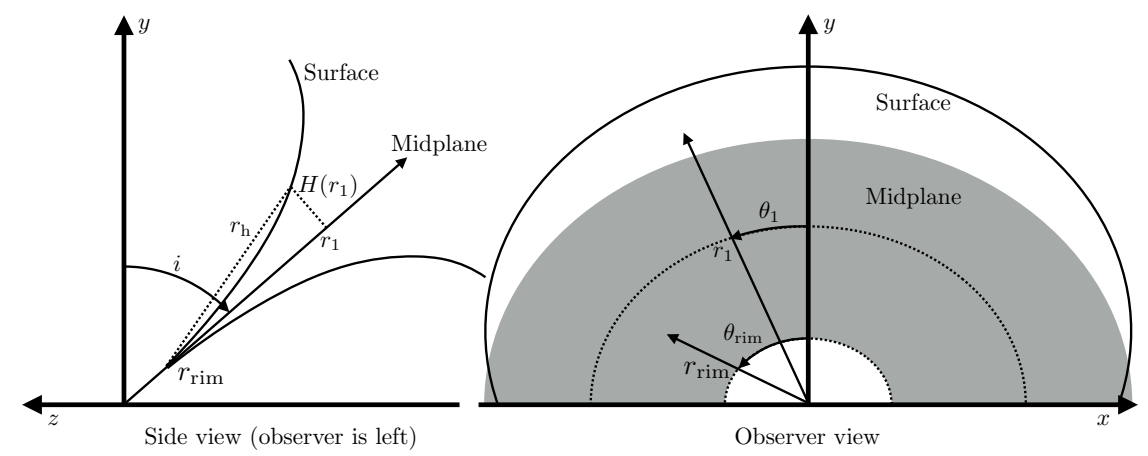

Fig. 2. Adopted parameterization for a flared and inclined disk.
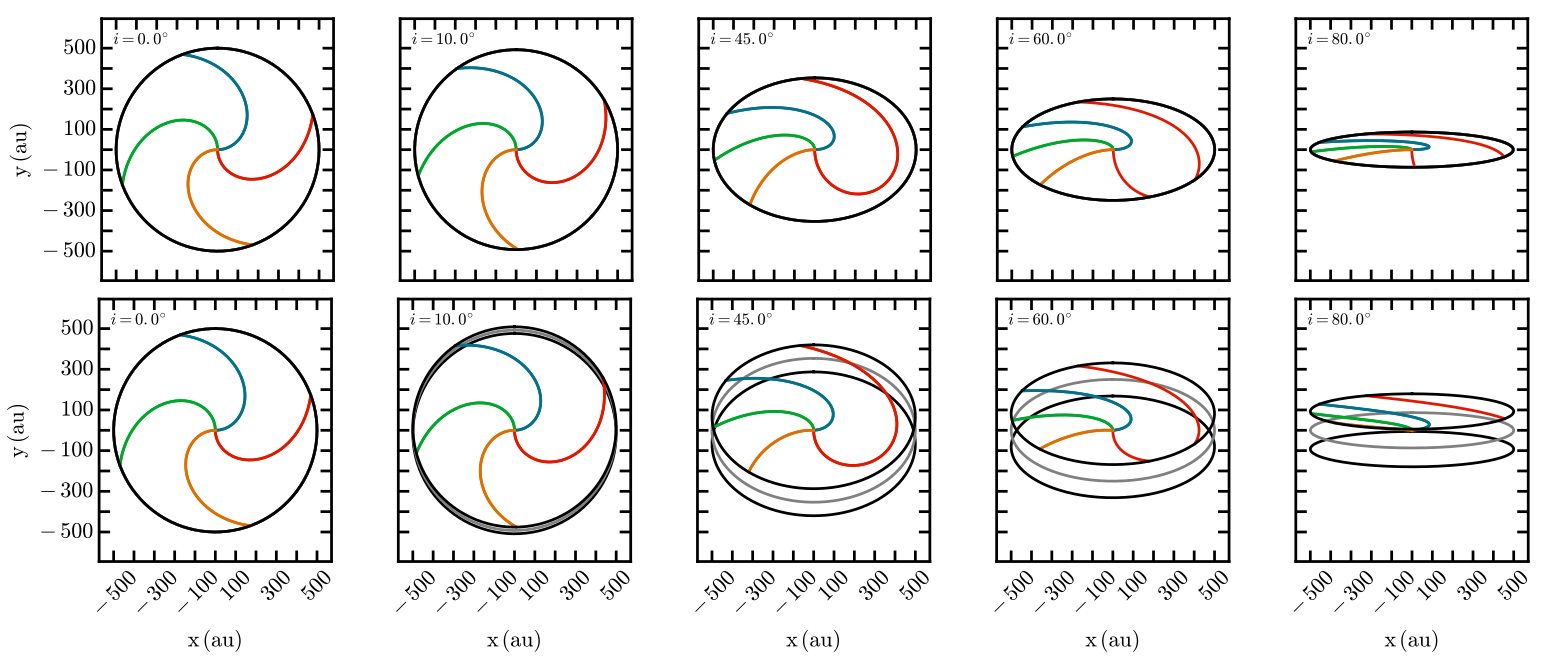

Fig. 3. Shadows cast on a flat (top panels) and flared (bottom) disk in clockwise rotation and with an outer radius of 400 au. The coloured curves indicate shadowing by clumps orbiting at $r_{\text {rim }}=0.1$ au and with instantaneous orbital phases of $\theta_{\text {rim }}=0, \pi / 2, \pi$, and $3 \pi / 2$ rad. The panels, from left to right, show $i=0^{\circ}, 10^{\circ}, 45^{\circ}, 60^{\circ}$, and $80^{\circ}$. Additional circles in the bottom row show the midplane and downward-facing edge of the disk.

and the top- and bottom-facing outer rim of the flared surface. The spirals are generally more tightly wound than for the flat disk, and differ more for higher inclinations.

In Fig. 4 the spirals are shown with a finite azimuthal width of $6 \%$ of $2 \pi$ at their origin $\left(r_{\text {rim }}=0.1 \mathrm{au}\right)$, for the case of $i=60^{\circ}$, to illustrate the shadow shapes for an obscuring clump of finite azimuthal size. Animations showing the shadows in motion for various inclinations are provided online, see Appendix A.

\section{Discussion}

\subsection{Comparison with other spiral-making processes}

Spirals in circumstellar disks may alternatively be formed from pressure waves induced when the disk interacts with itself, an orbiting planetary or stellar companion, or an external envelope; or by magnetohydrodynamical effects at the interface of highand low-ionisation regions within the disk (a so-called dead zone). The pattern speed of the spiral is controlled by the orbital angular-velocity at the spiral origin, proportional to $r^{-3 / 2}$ where $r$ is the radius of the inner-clump, planet, disk-envelope interface (Lesur et al. 2015), or ionisation gradient (Lyra et al. 2015).

Giant (proto-)planets capable of generating significant pressure waves are expected to be at radii of $10 \mathrm{~s}$ to $100 \mathrm{~s}$ of au, and the disk-envelope interface is at an even larger radius. Dead zones can give rise to azimuthally confined vortices at the inner thermal ionization boundary in a disk, corresponding to gas temperatures of $\sim 1000 \mathrm{~K}$, which is equivalent to radii $\sim 0.1$ au
(Umebayashi \& Nakano 1988; Desch \& Turner 2015). However, the pressure wave spirals originating from such regions are not sharply defined azimuthally or vertically at 10 s or 100 s of au, appearing as several tightly wound spiral arms (Baruteau \& Lin 2010; Lyra et al. 2015). The pattern speed of a delayed scatteredlight spiral caused by a shadowing clump at 0.1 au in the inner disk is probably a factor $\sim 1000$ greater than for a detectable pressure wave spiral.

Spiral curvature is proportional to the ratio of its pattern speed and radial propagation speed. As noted above, the pattern speeds of pressure wave spirals are expected to be significantly lower than for shadow spirals, but their radial propagation, approximately given by the speed of sound $\left(\sim 100-1000 \mathrm{~m} \mathrm{~s}^{-1}\right)$, is also reduced relative to the speed-of-light propagation for a shadow, by a factor of $\sim 10^{-6}$. Thus, a light travel time spiral will always be distinct from a pressure wave effect by its greater pattern speed, lesser curvature, or, more likely, both.

An important property of spirals arising from light travel time is that, unlike the limited parameter range of azimuthally asymmetric features caused by planets, self-gravity, infall envelopes, or Rossby wave instability, a large variety of structures can form, such as single, loosely-wound spiral arms and disjointed arcs, the shapes of which mainly depend on the location of a shadowing clump, disk aspect ratio, and disk inclination. These structures will change at the Keplerian time of the shadowing clump and with faster or slower variations in different parts of the disk due to inclination, as seen in Figs. 3 and 4. 


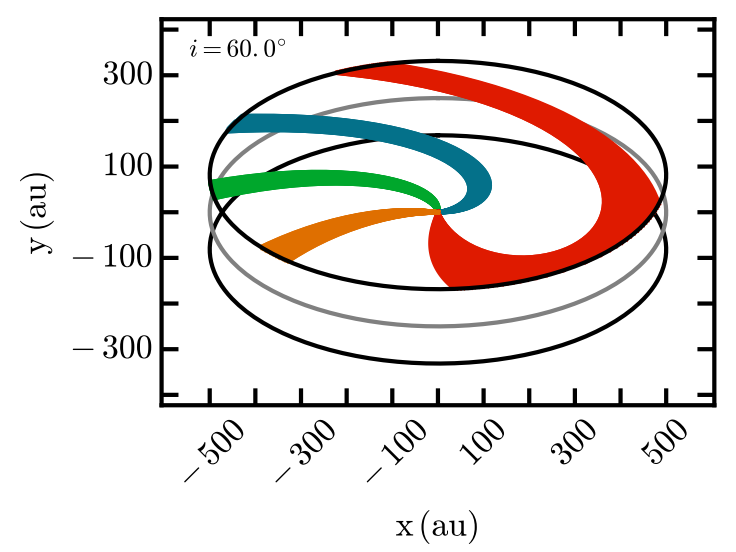

Fig. 4. Light travel time spirals for an inner disk perturbation shadowing (or, equivalently, illuminating) the disk across $6 \%$ of the azimuthal range. Solutions from Eq. (10) are plotted for a $1.5 M_{\odot}$ star and central origin points at $\theta_{\mathrm{rim}}=0, \pi / 2, \pi$, and $3 \pi / 2 \mathrm{rad}$.

\subsection{Partial shadowing: umbra and penumbra}

If the physical extent of the shadowing object is smaller than that of the central star, the shadow edge will no longer be well defined. There will be a radially converging full shadow (umbra) and a wider, longer partially shadowed region (penumbra). Our formulation assumes that the star is a point source, and therefore we cannot currently model such features. Should a (pen)umbra be observed, the physical dimensions of the shadowing object relative to those of the star will be strongly constrained, and the centre line of the penumbra will still correspond to Eq. (10).

\subsection{Observability}

Spatially resolved observations of faint and time-variable structures in scattered light have become feasible with recent advances in high-contrast, high angular resolution scattered-light imaging, brought about by the SPHERE and GPI instruments in particular. The spiral features predicted by Eq. (10) should be observable in such images. During the preparation of this manuscript, Stolker et al. (2016) reported a curved shadow in the HD 135344 B disk and determined that it is well fitted by the flat-disk shadow as described by Eq. (1). However, follow-up observations show that the feature has not moved on a timescale of about one month, inconsistent with the expected fast orbital timescale of a light travel time feature (Stolker, priv. comm.). In Subaru/HiCIAO $H$ - and $K$-band scattered-light imaging of four outbursting FU Ori disk systems, Liu et al. (2016) displayed a linear feature on a scale of 1000 au at an odd angle with respect to the centre-radial direction (their Fig. 1). The disk inclination is $i \approx 60^{\circ}$ (Malbet et al. 1993), and the observed feature resembles the theoretical red shadows shown in Fig. 3 for $i \approx 60^{\circ}$.

We have assumed an imageable scattered-light disk size of $500 \mathrm{au}$, which is larger than many disks currently accessible to imaging. For smaller disks, the full range of features shown in Fig. 3 will appear if the shadowing clump is closer to the star.

Given the rapidly increasing number of resolved, highcontrast disk images at visible and near-infrared wavelengths, and the potential for time-resolved multiple datasets, we are hopeful that the occurrence rate and morphological diversity of light travel time spirals will soon be robustly observationally tested. If they are common, a new tool will be available for constraining the inner and outer disk structure and distance to the systems. If light travel time spirals are not common, constraints can be set on the physical dimensions and survival timescales of major optical depth perturbations in the inner disk.

\section{Conclusions}

We have calculated the effect of light travel time on the shape of a shadow or a bright feature cast on a protoplanetary disk by a perturbation in the optical thickness of the inner disk for the general case of a flared and inclined disk. We find that the resulting scattered-light features can take on a range of curved shapes, in some cases forming disjointed and misaligned arcs.

We provide an expression (Eq. (10)) describing the shape of light travel time spirals in the general case that allows for easily predicting their form and for fitting of observations.

Fitting the equation to observed shadows allows the constraining of parameters such as the orbital motion of a shadowing clump, the mass of the central star, the outer disk vertical structure and physical scale, and the distance to the system. Recent scattered-light observations have found structures that resemble these phenomena and may provide a new way to study the structure and dynamics of material in the unresolved inner disk.

Acknowledgements. The authors thank Tomas Stolker and Wladimir Lyra for useful discussions. This work is supported by a Royal Netherlands Academy of Arts and Sciences (KNAW) professor prize.

\section{References}

Avenhaus, H., Quanz, S. P., Meyer, M. R., et al. 2014, ApJ, 790, 56 Baruteau, C., \& Lin, D. N. C. 2010, ApJ, 709, 759

Benisty, M., Juhasz, A., Boccaletti, A., et al. 2015, A\&A, 578, L6 Boccaletti, A., Pantin, E., Lagrange, A.-M., et al. 2013, A\&A, 560, A20 Bouvier, J., Alencar, S. H. P., Boutelier, T., et al. 2007, A\&A, 463, 1017 Burrows, C. J., Stapelfeldt, K. R., Watson, A. M., et al. 1996, ApJ, 473, 437 Desch, S. J., \& Turner, N. J. 2015, ApJ, 811, 156

Dong, R., Zhu, Z., Rafikov, R. R., \& Stone, J. M. 2015, ApJ, 809, L5 Dong, R., Fung, J., \& Chiang, E. 2016, ApJ, 826, 75 Garufi, A., Quanz, S. P., Schmid, H. M., et al. 2014, A\&A, 568, A40 Goldreich, P., \& Tremaine, S. 1979, ApJ, 233, 857

Grady, C. A., Polomski, E. F., Henning, T., et al. 2001, AJ, 122, 3396 Grady, C. A., Muto, T., Hashimoto, J., et al. 2013, ApJ, 762, 48 Hashimoto, J., Tamura, M., Muto, T., et al. 2011, ApJ, 729, L17 Juhász, A., Benisty, M., Pohl, A., et al. 2015, MNRAS, 451, 1147 Kenyon, S. J., \& Hartmann, L. 1987, ApJ, 323, 714

Lesur, G., Hennebelle, P., \& Fromang, S. 2015, A\&A, 582, L9 Liu, H. B., Takami, M., Kudo, T., et al. 2016, Sci. Adv., 2, e1500875 Lodato, G., \& Rice, W. K. M. 2004, MNRAS, 351, 630

Lyra, W., Turner, N. J., \& McNally, C. P. 2015, A\&A, 574, A10 Malbet, F., Rigaut, F., Bertout, C., \& Lena, P. 1993, A\&A, 271, L9 McCaughrean, M. J., \& O'dell, C. R. 1996, AJ, 111, 1977 McCaughrean, M. J., Chen, H., Bally, J., et al. 1998, ApJ, 492, L157 McGinnis, P. T., Alencar, S. H. P., Guimarães, M. M., et al. 2015, A\&A, 577, A11

Meng, H. Y. A., Plavchan, P., Rieke, G. H., et al. 2016, ApJ, 823, 58 Muto, T., Grady, C. A., Hashimoto, J., et al. 2012, ApJ, 748, L22 Ortiz, J. L., Sugerman, B. E. K., de La Cueva, I., et al. 2010, A\&A, 519, A7 Padgett, D. L., Brandner, W., Stapelfeldt, K. R., et al. 1999, AJ, 117, 1490 Pinilla, P., de Boer, J., Benisty, M., et al. 2015, A\&A, 584, L4 Rafikov, R. R. 2002, ApJ, 569, 997

Stauffer, J., Cody, A. M., McGinnis, P., et al. 2015, AJ, 149, 130

Stolker, T., Dominik, C., Avenhaus, H., et al. 2016, A\&A, submitted [arXiv: 1603.00481]

Umebayashi, T., \& Nakano, T. 1988, Prog. Theor. Phys. Suppl., 96, 151 Wagner, K., Apai, D., Kasper, M., \& Robberto, M. 2015, ApJ, 813, L2 Wisniewski, J. P., Clampin, M., Grady, C. A., et al. 2008, ApJ, 682, 548 
M. Kama et al.: Spirals in protoplanetary disks from photon travel time

\section{Appendix A: Videos of light travel time spirals}

We provide videos of solutions to Eq. (10), showing spiral- and arc-shaped shadows rotating on the Keplerian timescale of the clump casting the shadow at $r_{\text {rim }}=0.1$ au around a $1.5 M_{\odot}$ star. Three inclinations are considered: $i=0^{\circ}, 30^{\circ}$, and $60^{\circ}$.

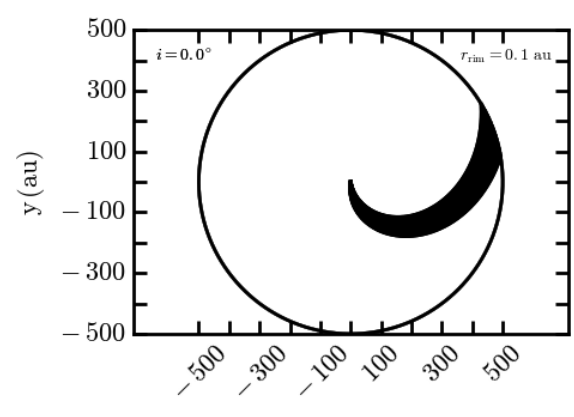

$\mathrm{x}(\mathrm{au})$

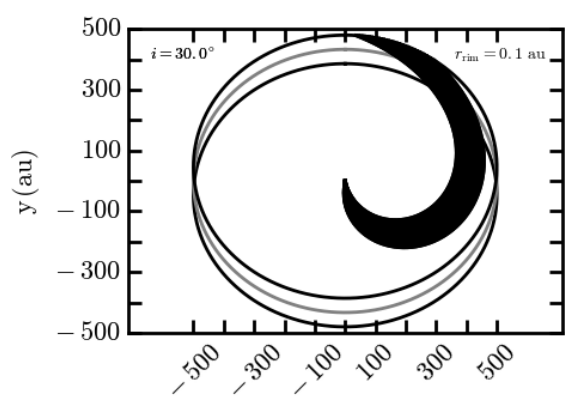

$\mathrm{x}(\mathrm{au})$

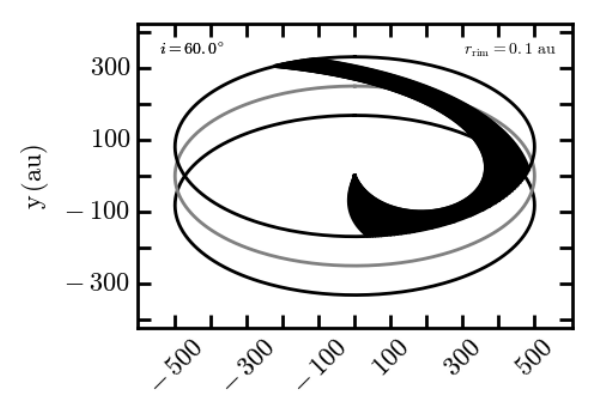

$\mathrm{x}(\mathrm{au})$

Fig. A.1. Light travel time spiral solutions from Eq. (10), originating at a shadowing clump at $r_{\text {rim }}=0.1$ au around a $1.5 M_{\odot}$ star. The panels show different inclinations - left panel: $i=0^{\circ}$ (movie 1), middle panel: $30^{\circ}$ (movie 2), right panel: $60^{\circ}$ (movie 3 ). The instantaneous orbital phase of the video snapshots is $\pi \mathrm{rad}$. 